\title{
Simulation AND ANALYSis OF Cognitive RADio SYSTEM USING MATLAB
}

\author{
Goutam Ghosh $^{1}$, Prasun Das ${ }^{2}$ and Subhajit Chatterjee ${ }^{3}$ \\ ${ }^{1}$ Department of Electronics and Communication Engineering, College of \\ Engineering and Management,Kolaghat, West Bengal, India \\ ${ }^{2}$ Department of Electronics and Communication Engineering, Bengal Institute \\ of Technology and Management,Santiniketan, West Bengal, India \\ ${ }^{3}$ Department of Electronics and Communication Engineering, Swami \\ Vivekananda Institute of Science and Technology, Kolkata, West Bengal, India
}

\begin{abstract}
The increasing demand of wireless applications has put a lot of limitations on the use of available radio spectrum is limited and precious resource. Many survey of spectrum utilization shows that entire spectrum is not used at all the times, so many of the radio spectrum is underutilized. Some of the frequency bands in the spectrum are unoccupied, some of the frequency bands less occupied and few bands are over utilized. Cognitive radio system is a technique which overcomes that spectrum underutilization. Cognitive radio is a technique where secondary user looks for a free band to use when primary user is not in use of its licensed band. A function of cognitive radio is called Spectrum sensing which enables to search for the free bands and it helps to detect the spectrum hole (frequency band which is free enough to be used) which can be utilized by secondary user with high spectral resolution capability. The idea of simulation and analysis of Cognitive Radio System to reuse unused spectrum to increase the total system capacity was brought in this paper and this work digs into the practical implementation of a Cognitive radio system. MATLAB R2007b (version7.5) has been used to test the performance of Cognitive radio dynamically.
\end{abstract}

\section{KEYWORDS}

Cognitive Radio, Spectrum Sensing, Energy detection and Periodogram, MATLAB

\section{Introduction}

The wide growth of wireless communications leads to the scarcity of frequency spectra and available radio spectrum is a limited natural resource, being congested day by day. Many of the preallocated frequency bands are ironically under-utilized, the resources there are simply wasted. It has been found that the allocated spectrum is underutilized for static allocation of the spectrum. 


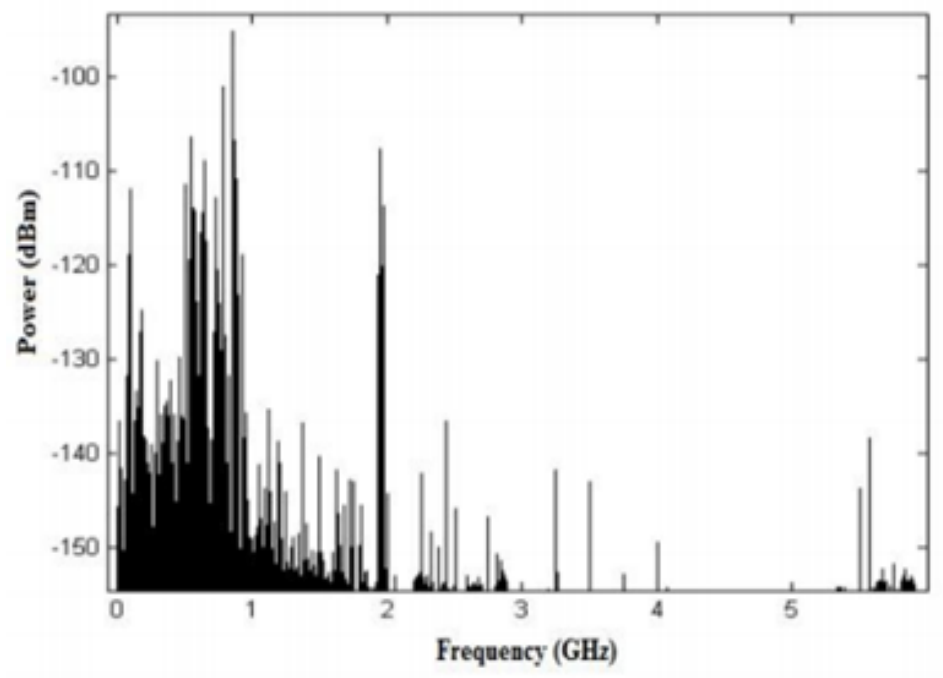

Figure 1. Graph of spectrum utilization at Berkeley Wireless Research Centre

The conventional approach to spectrum management is not flexible. To operate, every wireless operator is assigned a sole license in a certain frequency band. It is difficult to find vacant bands to deploy new services and enhance existing ones. To overcome this situation, we need a improved utilization of the spectrum which will create opportunities for Dynamic Spectrum Access (DSA). A possible solution is the use of "Cognitive Radio" technology which is a radio or system, it has the ability to sense and is fully aware of its functioning condition and can regulate its operating parameters. This technique seems like a promising solution for the use of available spectrum on the frequency band efficiently. By analyzing, observing and learning, the Cognitive radio adapts to the environment conditions and makes use of this analysis for future decisions. There are mainly two tiers of users in the cognitive radio model. Primary Users (PU) are licensed users, have the rights of priority in using certain stable frequency band for communications, Secondary Users (SU) are allowed to use the frequency spectra momentarily only if they do not interfere with the PU. So the ability of sensing an idle spectrum and the ability to temporarily utilize a spectrum without interfering with Primary Users are two essential components required for the success of cognitive radios [1].

In this paper, Section 1 gives introduction about the cognitive radio and Section 2 contains detailed definition of cognitive radio, Spectrum sensing techniques have been explained in section 3, section 4 shows Methodology for Implementation of Cognitive Radio System, Results are shown in section 5 and section 6 concludes the discussion.

\section{Cognitive Radio}

Cognitive radio is a form of wireless communication where a transceiver can intelligently detect the channels for communication which are in use and which are not in use, and move into unused channels while avoiding occupied ones. This optimizes the use of available radio-frequency spectra while interference is minimized to other users. This is a paradigm for wireless communication where transmission or reception parameters of network or node are changed for communication avoiding interference with licensed or unlicensed users. A spectrum hole (Figure 2) is generally a concept of spectrum as non-interfering, considered as multidimensional areas within frequency, time, and space. For secondary radio systems, the main challenge is to be able to sensing spectrum hole [2] when they are within such frequency bands. 


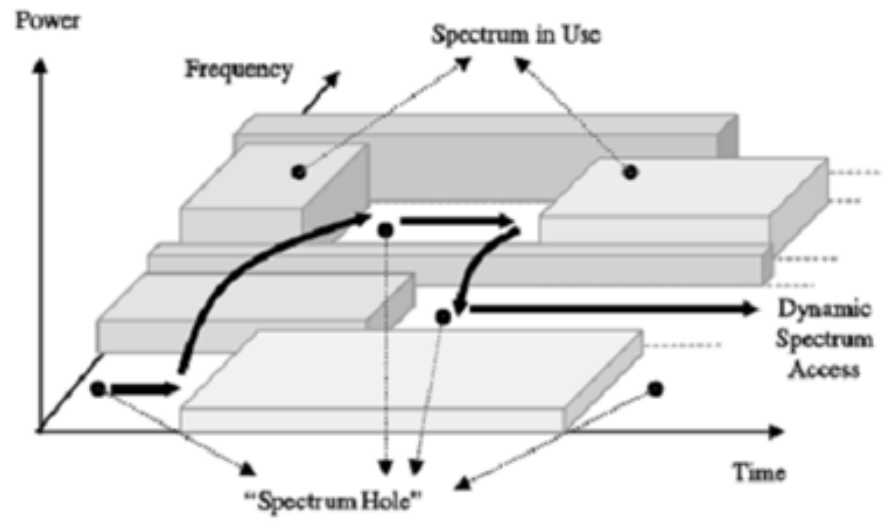

Figure 2. Spectrum holes concept

\subsection{Types of $\mathbf{C R}$}

There are two types of Cognitive Radios:

- Full Cognitive Radio: Full Cognitive Radio (CR) considers all parameters. A wireless node or network can be conscious of every possible parameter observable [3].

- Spectrum Sensing Cognitive Radio: Detects channels in the radio frequency spectrum. Fundamental requirement in cognitive radio network is spectrum sensing. To enhance the detection probability [4] many signal detection techniques are used in spectrum sensing.

The performances for cognitive radio system requires: i) authentic spectrum hole and detection of primary user, ii) precise link estimation between nodes, iii) fast and accurate frequency control and iv) method of power control that assures reliable communication between cognitive radio terminals and non-interference to the primary users [3].

\subsection{Characteristics of $\mathbf{C R}$}

There are two main characteristics [5] of the cognitive radio and can be defined

- Cognitive capability: Cognitive Capability defines the ability to capture or sense the information from its radio environment of the radio technology. Joseph Mitola first explained the cognitive capability in term of the cognitive cycle "a cognitive radio continually observes the environment, orients itself, creates plans, decides, and then acts"

- Reconfigurability: Cognitive capability offers the spectrum awareness, Reconfigurability refers to radio capability to change the functions, enables the cognitive radio to be programmed dynamically in accordance with radio environment (frequency, transmission power, modulation scheme, communication protocol).

\subsection{Functions of $\mathbf{C R}$}

There are four major functions of Cognitive Radio. Figure 3. shows the basic cognitive cycle 


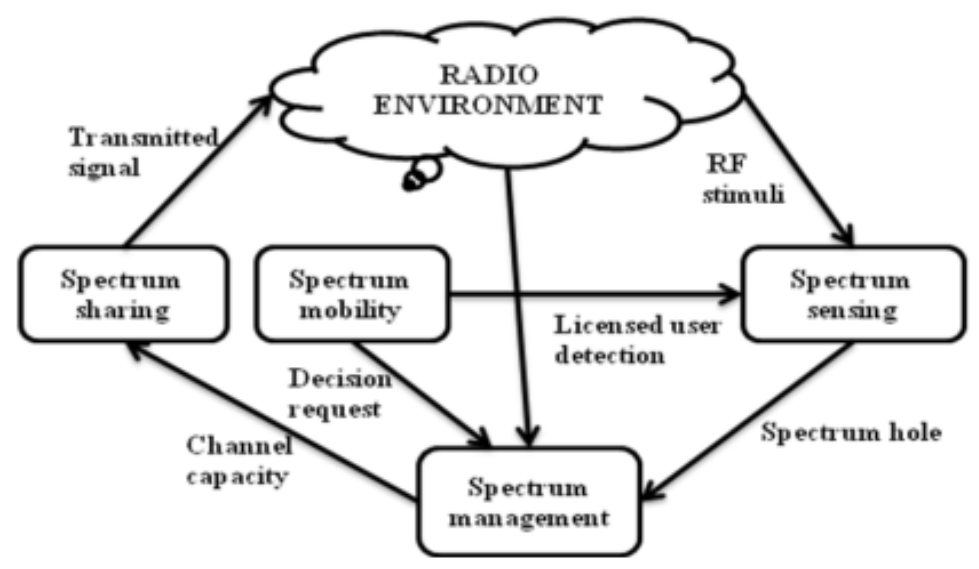

Figure 3. Basic cognitive cycle

\subsubsection{Spectrum Sensing}

The first step of spectrum sensing is that it determines the presence of primary user on a band. The cognitive radio is able to share the result of its detection with other cognitive radios after sensing the spectrum [6]. The goal of spectrum sensing is to find out the spectrum status and activity by periodically sensing the target frequency band. Particularly, a cognitive radio transceiver detects the spectrum which is unused or spectrum hole and also determines method of access without interfering the transmission of licensed. Two types of spectrum sensing are there; it may be either centralized or distributed. In the centralized spectrum sensing, a sensing controller senses the target frequency band, and share the information with other nodes in the system.

\subsubsection{Spectrum management}

Spectrum Management: Provides the fair spectrum scheduling method among coexisting users. The available white space or channel is immediately selected by cognitive radio if once found. This property of cognitive radio is described as spectrum management. Spectrum sensing, spectrum analysis, and spectrum decision fall in spectrum Management. Spectrum Sensing has been discussed in previous section. Spectrum Analysis makes possible the characterization of different spectrum bands, which is exploited to get the spectrum band appropriate requirements of the user. Spectrum decision refers to a cognitive radio decides the data rate, determines the transmission mode, and the transmission bandwidth. Then, the appropriate spectrum band is selected according to the spectrum characteristics and user requirements.

\subsubsection{Spectrum Sharing}

Cognitive Radio assigns the unused spectrum (spectrum hole) to the secondary user (SU) as long as primary user (PU) does not use it. This property of cognitive radio is described as spectrum sharing.

- Underlay Spectrum Sharing: Underlay spectrum sharing is the availability of the radio spectrum access with minimal transmission power that the interference temperature 
International Journal of Next-Generation Networks (IJNGN) Vol.6, No.2, June 2014 above its pre-designed thresholds wouldn't be raised. To spread the unlicensed signal over a large band of spectrum in underlay spectrum sharing the licensed radio device can identify undesired signal which is below the noise and interference floor[7].

- Overlay Spectrum sharing: Unlicensed users can utilize a spectrum band for the fraction of time where this band is under-utilized by the licensed users in Overlay Spectrum sharing technique.

\subsubsection{Spectrum Mobility}

When a licensed (Primary) user is detected the Cognitive Radio (CR) vacates the channel. This property of cognitive radio is described as the spectrum mobility and also called handoff [8]. This is the process that allows the Cognitive Radio user to change its operating frequency. Cognitive Radio networks try to use the spectrum dynamically to operate in the best available frequency band and maintain the transparent communication. Spectrum sensing is an important and a sensitive job out of these four functions in Cognitive Radio since interfering with other users is illegal.

\section{SPECTRUM DETECTION TECHNIQUES}

Many different methods are proposed to identify the presence of signal transmission and can be used to enhance the detection probability.

\subsection{Energy detection}

The aim of the spectrum sensing is to decide between two hypotheses which are
$\mathrm{x}(\mathrm{t})=\mathrm{w}(\mathrm{t}), \mathrm{H}_{0}$
(Primary User absent)
$x(t)=h n(t)+w(t), H_{1}$
(Primary User present)

Where $x(t)$ is the signal received by the CR user, $n(t)$ is the transmitted signal of the primary user, $\mathrm{w}(\mathrm{t})$ is the AWGN band, $\mathrm{h}$ is the amplitude gain of the channel. $\mathrm{HO}$ is a null hypothesis, which states that there is no licensed user signal.

Energy Detection is the common way of spectrum sensing for its low computational and implementation complexities. It is a non coherent detection method which is used to detect the licensed user signal [9] and is based on the use of the FFT (Fast Fourier transform), which transforms a signal from a time domain to a frequency domain representation, determines the power in each frequency of the signal resulting in which is known as the PSD (Power Spectral Density).

In this technique, the output of the energy detector compares with a threshold depending on the noise floor and signal is detected.

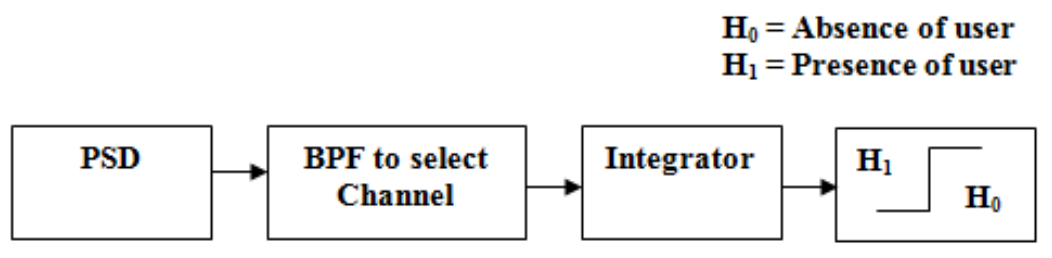

Figure 4. Block diagram of energy detector 
Figure 4 shows block diagram of energy detector [10]. Here signal is applied to band pass (BP) filter to select channel and is integrated over time interval. Lastly the output of the integrator compares with a threshold to determine whether primary user is present or not. The threshold value can set to be fixed or variable based on the channel conditions.

\subsection{Matched filter detection}

The matched filter referred to as coherent detector, and is shown in Figure 5. The probability of error in elementary communication theory [11] for a coherent detector and White Gaussian noise statistics is:

$P_{e}=Q\left(\frac{\sqrt{E_{s}}}{2 \sigma}\right)$

where Es is the energy in the LO signal, $\sigma 2$ is the receiver noise power, and $\mathrm{Q}(\mathrm{x})=1-$ $\mathrm{F}(\mathrm{x})$, where $\mathrm{F}(\mathrm{x})$ is the standard normal distribution function.

This is an optimal detector in Gaussian noise which maximizes the received signal-tonoise ratio (SNR). Matched filter determines the presence of the PU by correlating the signal with time shifted version and comparing between the predetermined threshold and output of matched filter.

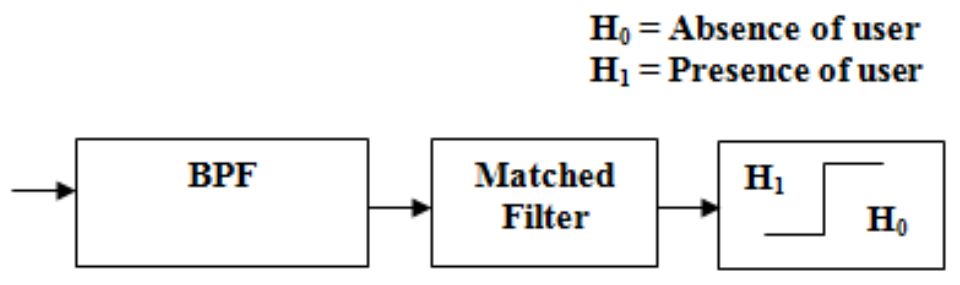

Figure 5. Block diagram of matched filter

The matched filter detection operation is expressed as [12]:

$\mathrm{Y}(\mathrm{n})=\sum_{\mathrm{k}=\infty}^{\infty} \mathrm{h}(\mathrm{n}-\mathrm{k}) \mathrm{x}(\mathrm{k})$

Where ' $\mathrm{x}$ ' is the unknown signal convolved with the ' $h$ ' (impulse response of matched filter which is matched to the reference signal) to maximize the SNR. This is useful for detection only when the information from primary users (PU) is known to cognitive users. Some drawbacks of this technique which are: (i) A prior knowledge of every primary signal are required. (ii) A dedicated receiver is needed for every type of cognitive primary user.

\subsection{Cyclostationary Feature Detection}

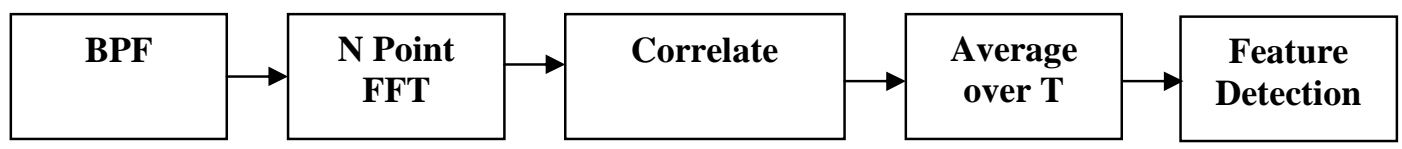

Figure 6. Block diagram of Cyclostationary feature detector 
International Journal of Next-Generation Networks (IJNGN) Vol.6, No.2, June 2014

Cyclostationary feature detection based on introduction of periodic redundancy into a signal by sampling and modulation. The periodicity in the received primary signal to identify the presence of Primary Users (PU) is exploited by Cyclostationary feature detector [13] which measures property of a signal namely Spectral Correlation Function (SCF) given by

$S_{x}^{\infty}(f)=\int_{-\infty}^{\infty} R_{x}^{\infty}(\tau) e^{-j 2 \pi f \tau} d \tau$

Where $R_{x}^{\infty}(\tau)$ is cyclic autocorrelation function (CAF).

Cyclostationary feature detector implementation can differentiate the modulated signal from the additive noise, distinguish Primary User signal from noise. It is used at very low SNR detection by using the information embedded in the Primary User signal which does not exist in the noise.

This technique is robust to noise discrimination and it performs better than energy detector. It has disadvantage of more computational complexity and longer time observation.

\subsection{Cooperative detection}

Sensing will be accomplished by a number of different radios within a cognitive radio network in a cooperative cognitive radio spectrum sensing system. Typically reports of signals from different radios in the network are received by a central station which concludes their combined decision by some particular fusion rule.

The operation of this technique is performed as follows [14]:

Step 1: Local spectrum measurements are performed independently by every cognitive radio and then made a binary decision.

Step 2: Binary decisions of all the cognitive radios are forwarded to a common receiver i.e., an access point (AP) in a WLAN or a BS (Base Station) in a cellular network.

Step 3: Those binary decisions are combined by the common receiver and made a final decision to infer the presence or absence of the primary user (PU) in the band observed.

\subsection{Interference-based detection}

Interference occurs at receivers, in trans-centric way it is regulated and is controlled at the transmitter through the location of individual transmitters and radiated power. A model of Interference temperature is shown in Figure 7 [15]. 


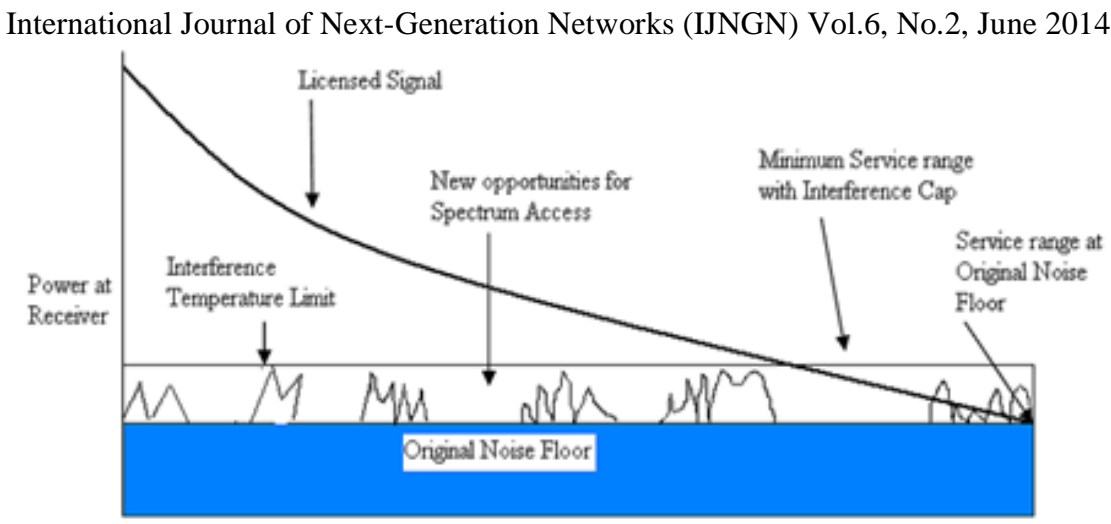

Figure 7. Interference temperature model

The working principle of this technique is like an UWB technology, when the CR users are allowed to coexist and transmit simultaneously with primary users (PU) using low transmit power is restricted by the interference temperature level as a result no harmful interference to primary users does not occur.

\section{Methodology for Cognitive Radio System Implementation USING MATLAB}

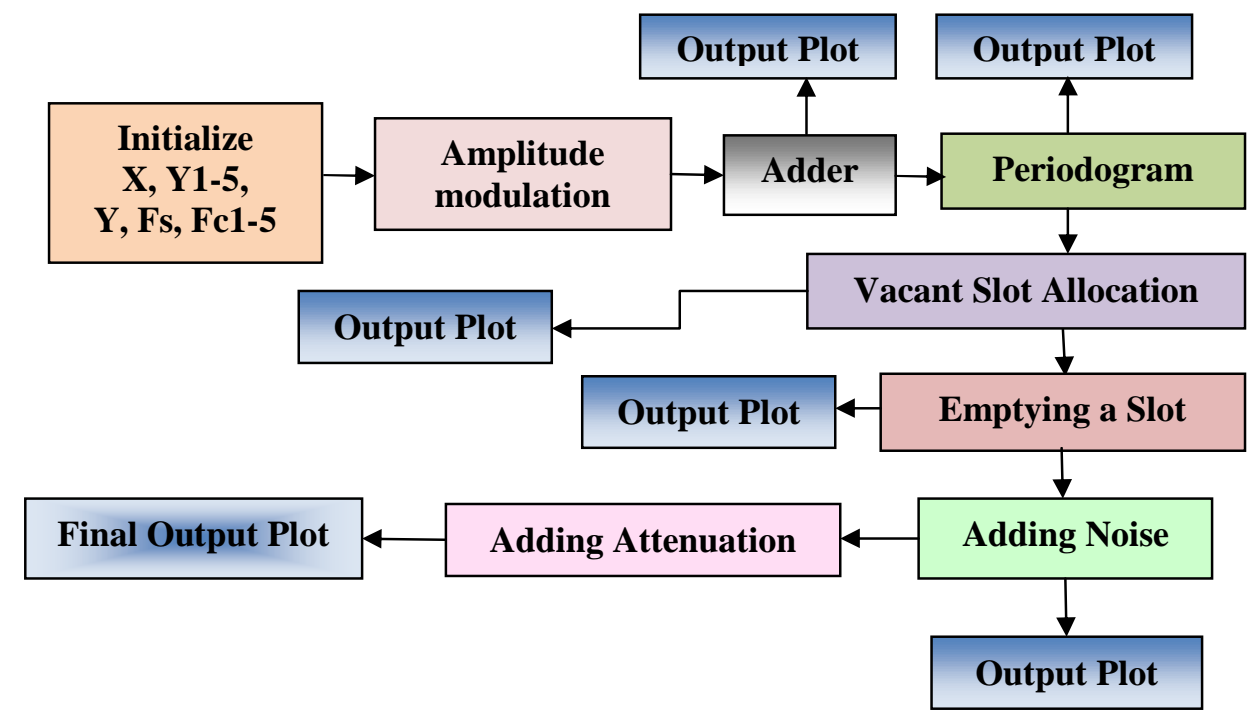

Figure 8. Methodology/Block diagram of simulation set up.

- Initialization- 5 Carrier Frequency Bands for Users, Message Frequency and the Sampling Frequency are initialized.

- Modulation- Modulates user data over the respective frequency band by amplitude modulation

- Adder- Addition of all the modulated signals to produce a transmitting signal

- Periodogram- To estimate the power spectral density of received signal.

- Vacant Slot Allocation- New User is allotted to the first spectral hole when he arrives.

- Emptying a slot- Asked user to empty a specific slot if all the slots are engaged. 
International Journal of Next-Generation Networks (IJNGN) Vol.6, No.2, June 2014

- Addition of noise- Amount of Noise to be added

- Attenuation- Percentage of Attenuation is introduced

\section{RESULT}

The cognitive radio system continuously searches the spectrum hole where primary user is not present and is determined by the method of energy detection. When it finds out the spectrum hole, immediately it allots to the Secondary User (SU) and whenever Primary User (PU) wants to occupy the slot, Secondary User immediately leaves it [16].

For 5 (Five) signals, the carrier frequencies are $1 \mathrm{MHz}, 2 \mathrm{MHz}, 3 \mathrm{MHz}, 4 \mathrm{MHz}, 5 \mathrm{MHz}$ and sampling frequency is $12 \mathrm{MHz}$ used for simulation. Power Spectrum Density (PSD) of signal is calculated, compared with the predefined threshold value and determined the presence of primary user signal.

In this paper, it has assumed that 1st, 5th primary users are present and $2 \mathrm{nd}$, 3rd and 4th primary users are not present. Then, the following results are obtained which are shown in the Figure 9.(a), Figure 9.(b), Figure 9.(c), Figure 9.(d) and . Figure 9. (e)

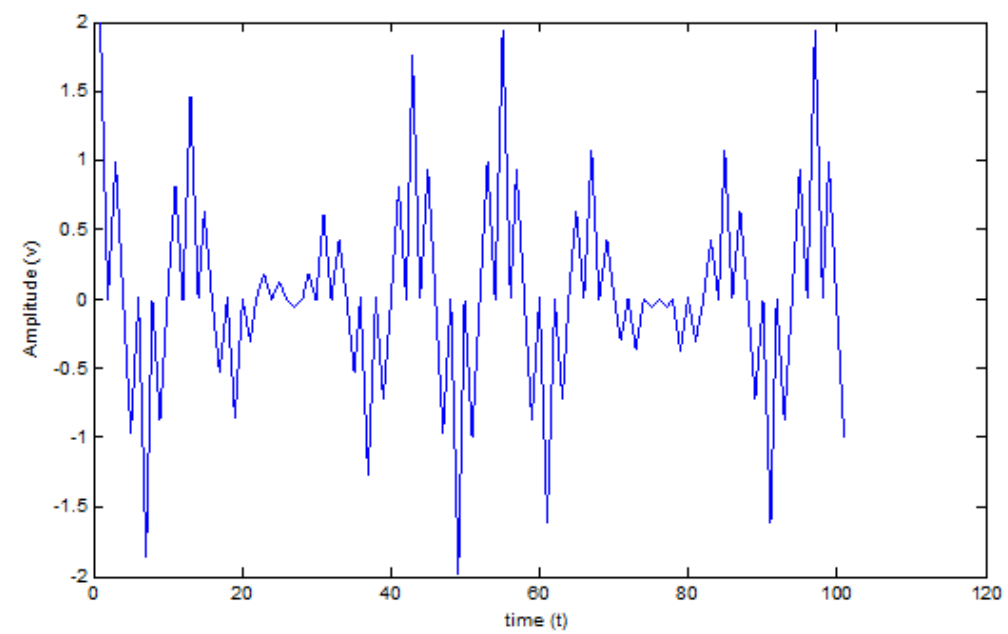

Figure 9. (a) Adder Output. User Present (1st and 5th), User Absent (2nd, 3rd, and 4th)

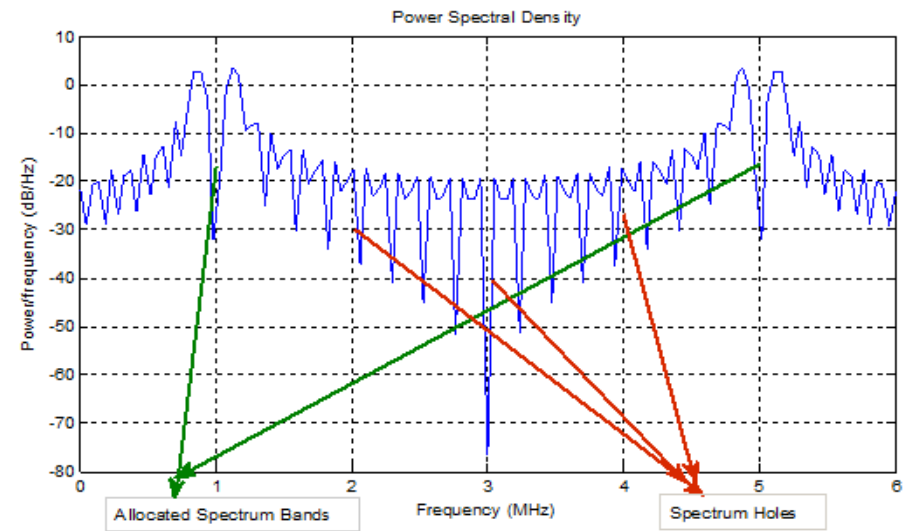

Figure 9. (b) Used bands (1st and 5th), Unused bands (2nd, 3rd, and 4th) 
International Journal of Next-Generation Networks (IJNGN) Vol.6, No.2, June 2014

Now the Cognitive Radio (CR) system will look for the first available gap (Spectrum hole) and automatically assign it to the secondary user (SU) in the spectrum. It is shown in the Figure 9. (c) that the first available spectral gap was occupied by the secondary user (SU) 1 .

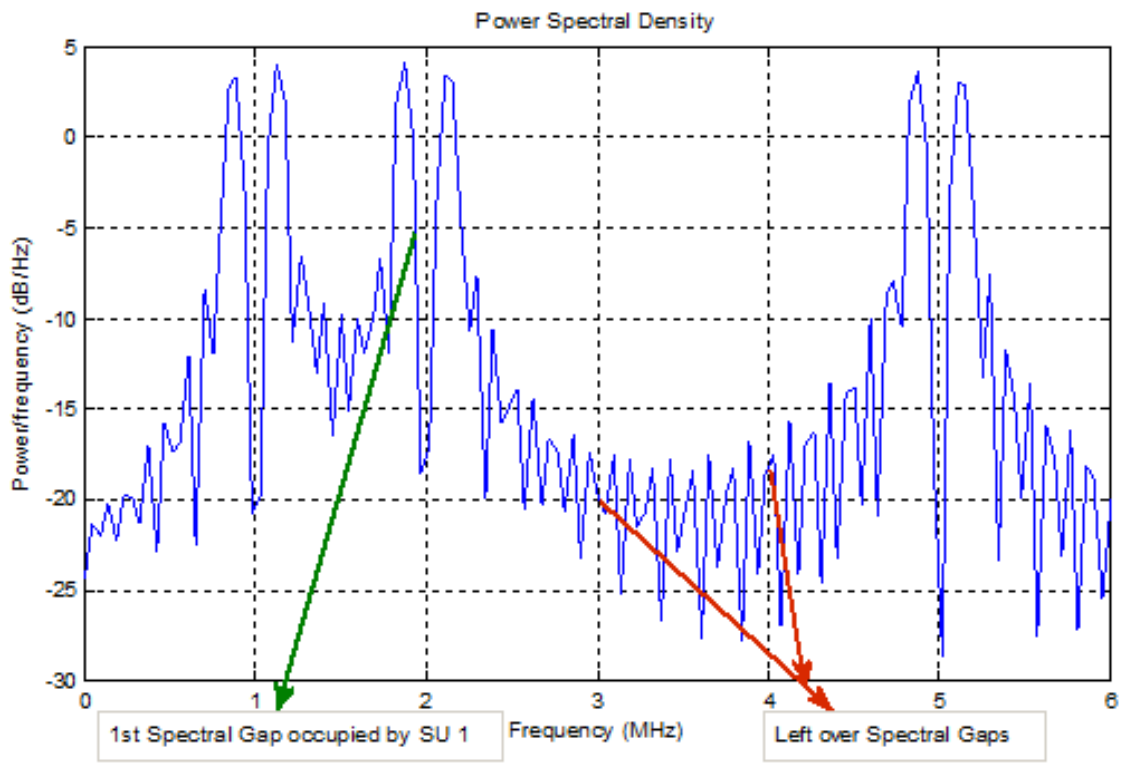

Figure 9. (c) 1st unused band assigned to Secondary User 1

Now the system will search the next spectrum hole and automatically assign it to the secondary user (SU) in the spectrum. As shown in the Figure 9. (d), the next available gap was occupied by the secondary user (SU) 2 .

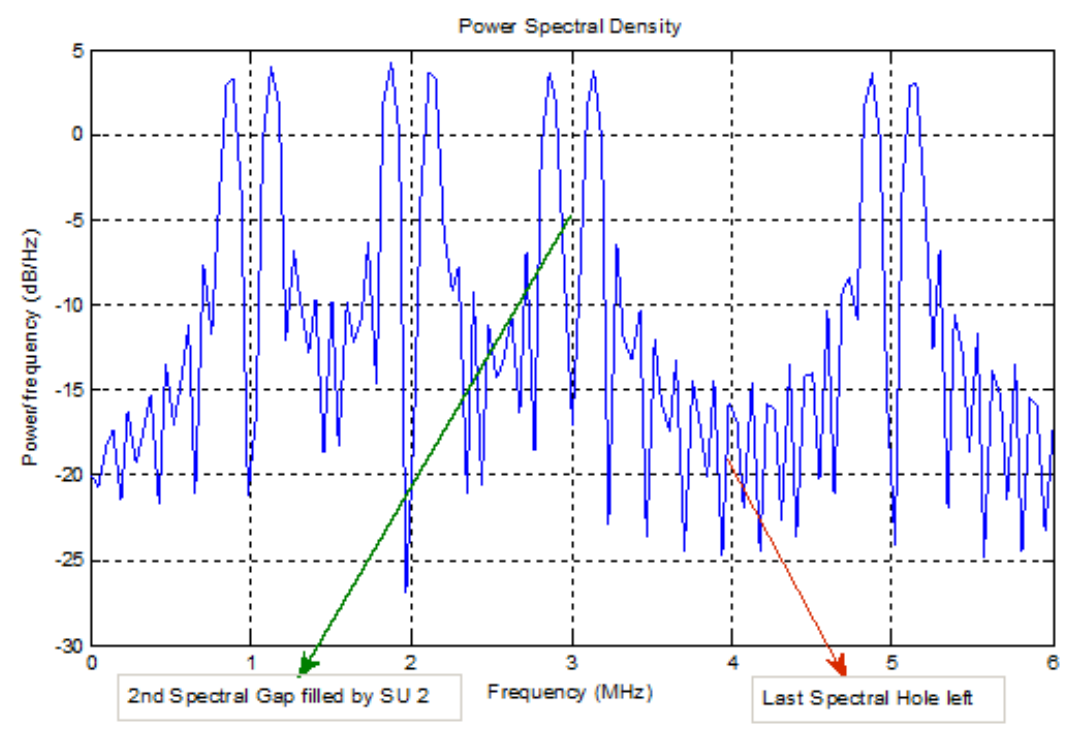

Figure 9. (d) 2nd unused band assigned to Secondary User 2 


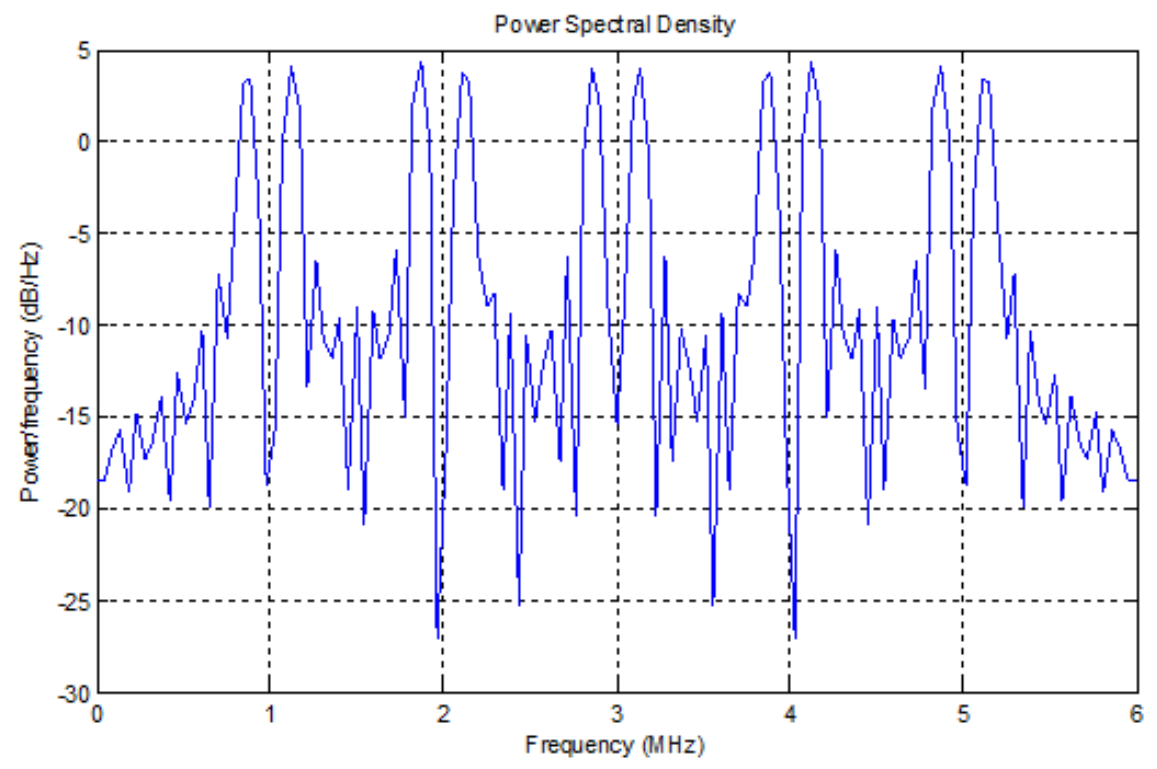

Figure 9. (e) All of the Spectrum bands are in use

Now just one slot left empty which will get filled by addition of another Secondary User (SU) as shown in Figure 9. (e). Here all of the frequency bands are in use efficiently after the last spectrum hole is filled by secondary user 3 .

Low peaks in Figure 9. (b) are for 2nd, 3rd and 4th primary users who are not present and high peaks for the 1 st and 5th primary user (PU) who are present. It is seen in Figure 9. (c) that there is an increase in the peak of 2nd slot after allocating the 2nd slot to secondary user1. Similarly in Figure 9. (d), an increase in the peak of 3rd slot allocating it to the secondary user (SU) 2 is observed. At this instant 4th primary user leaves the slot. So, finally, the allotment of 4th slot to secondary user 3 by the cognitive radio network is seen Figure 9. (e).

Once all the slots are being assigned, system will not entertain other user and will be able to free up the spectral gap (slot) one by one. If asked to empty a slot, it will delete the data in the first spectral gap and make it ready for the next assignment.

To analyze the channel characteristics we can add noise and attenuation parameter. Now if the Signal to Noise Ratio (SNR) is $5 \mathrm{~dB}, 15 \mathrm{~dB}$. Then, the following results are shown in the Figure 9.(f) and Figure 9.(g). The disturbance in the spectrum can be observed to decrease with the increase in SNR. This means that the noisy channel will increase the probability of error in the received signal. 
International Journal of Next-Generation Networks (IJNGN) Vol.6, No.2, June 2014

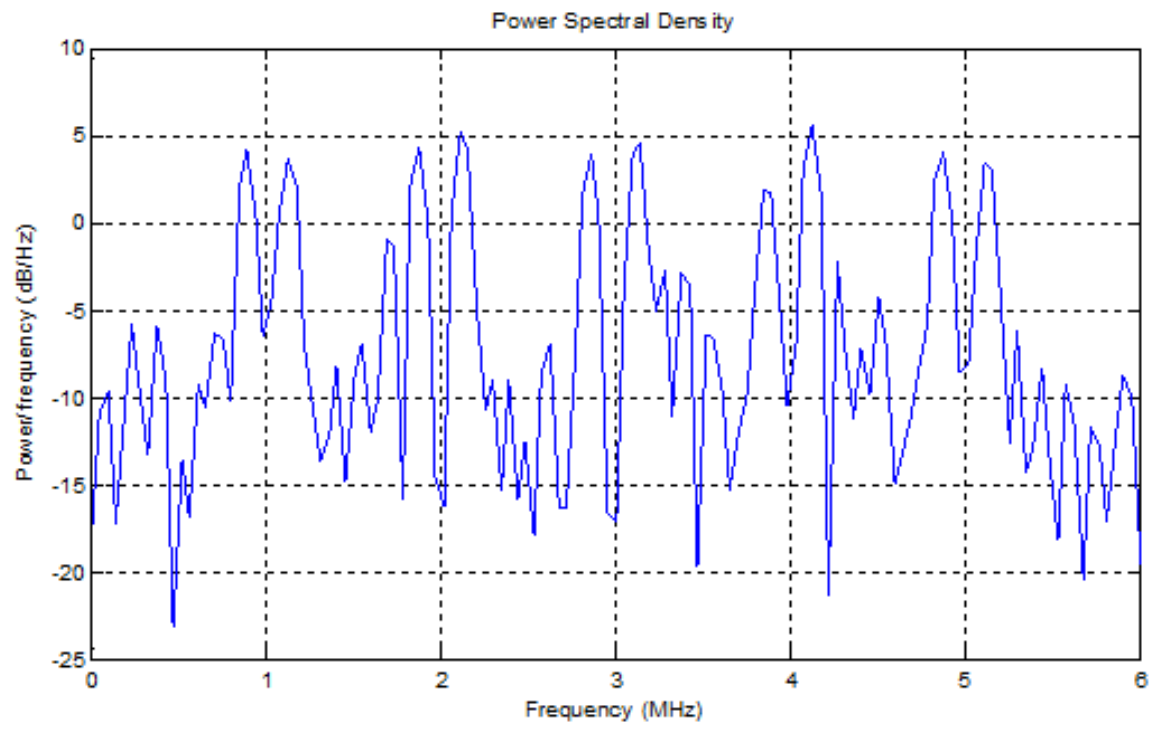

Figure 9. (f) $\mathrm{SNR}=5 \mathrm{~dB}$

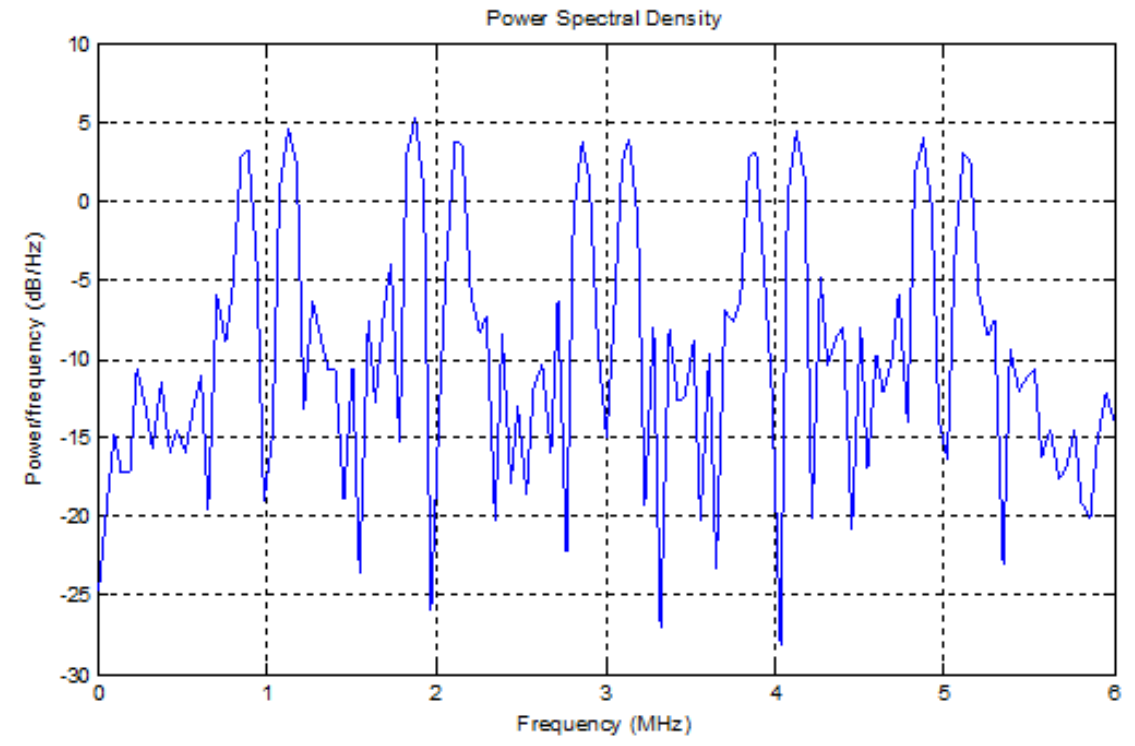

Figure 9. (g) $\mathrm{SNR}=15 \mathrm{~dB}$

If the attenuation percentage values $10 \%$ and $15 \%$ are taken, then the following results are observed in the Figure 9. (h) and Figure 9. (i). Here the signal peaks are proportionately reduced with increasing attenuation thus attenuation in the channel will reduce the signal power which in essence impairs the proper signal reception. 
International Journal of Next-Generation Networks (IJNGN) Vol.6, No.2, June 2014

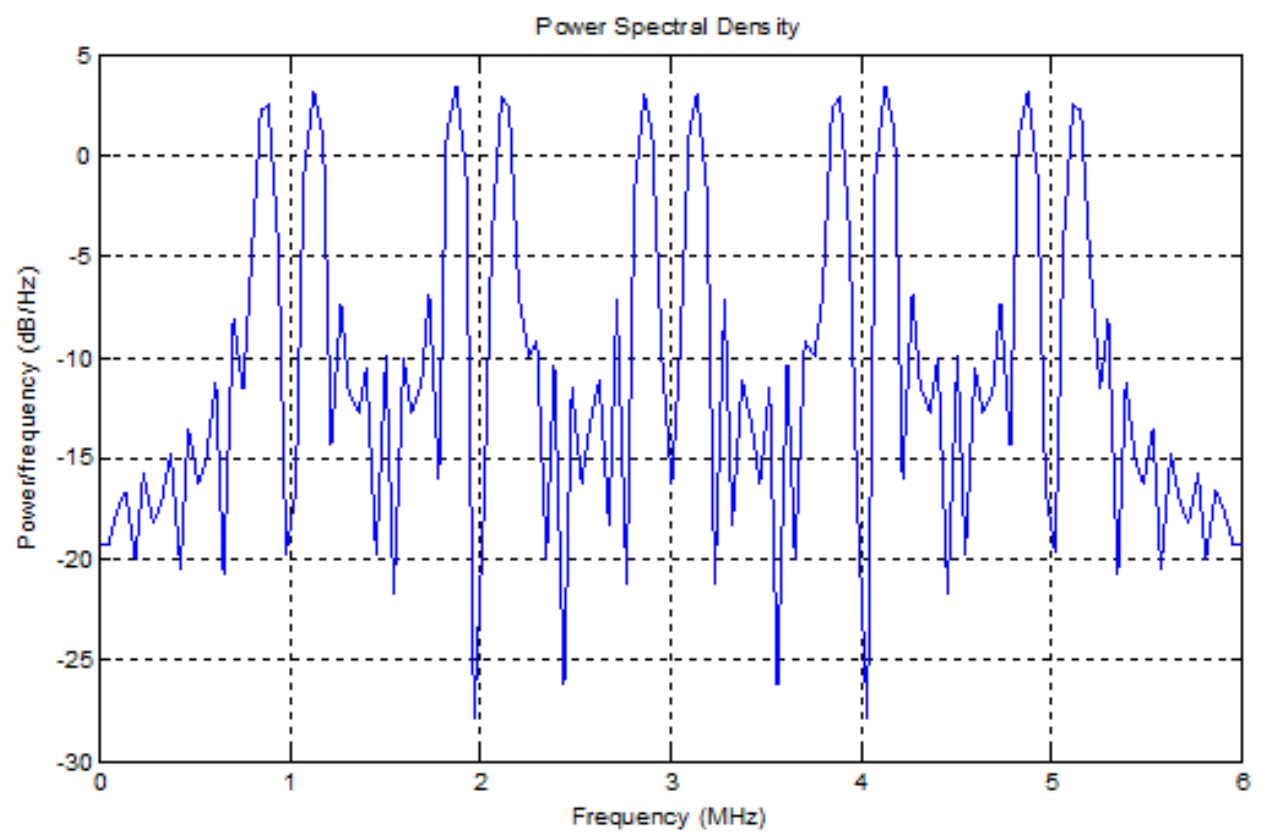

Figure 9. (h) Attenuation $=10 \%$

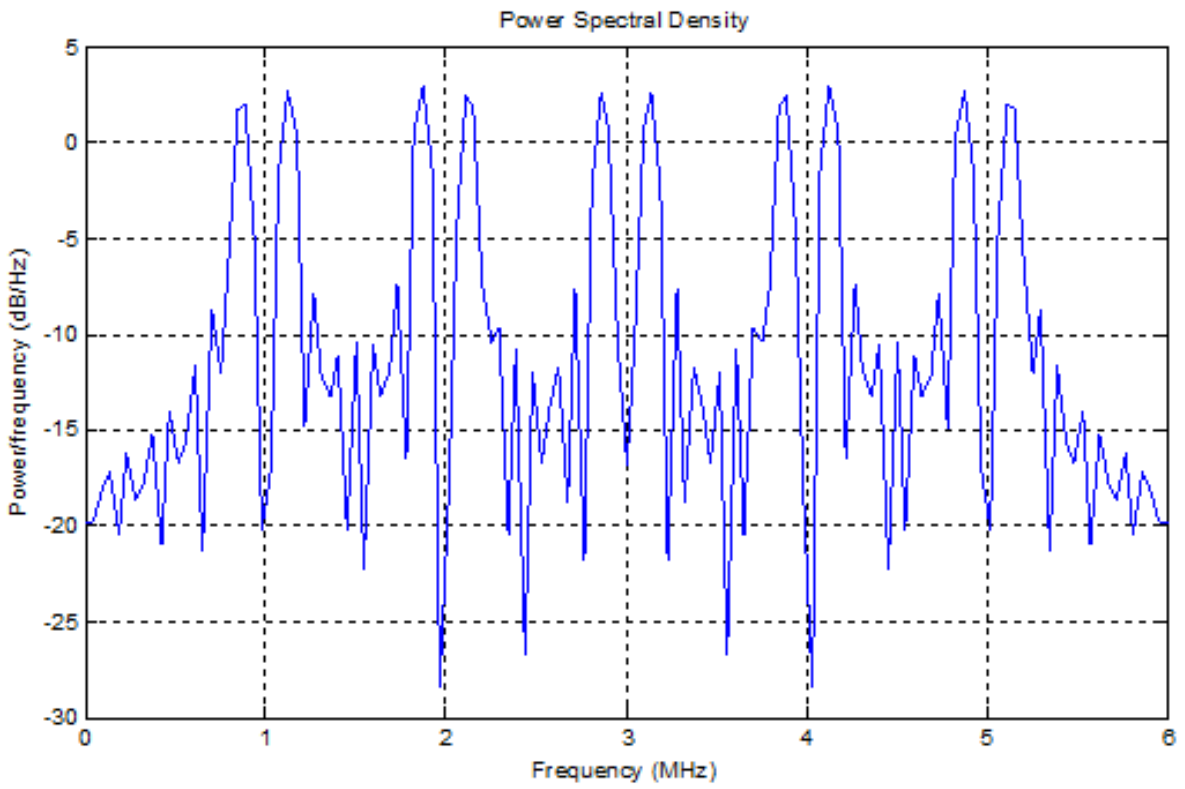

Figure 9. (i) Attenuation $=15 \%$

The simulation can also be carried out with the following combination of parameters:

$\mathrm{SNR}=20 \mathrm{~dB}$ and Attenuation $=15 \%$

$\mathrm{SNR}=25 \mathrm{~dB}$ and Attenuation $=10 \%$

$\mathrm{SNR}=20 \mathrm{~dB}$ and Attenuation $=20 \%$

$\mathrm{SNR}=20 \mathrm{~dB}$ and Attenuation $=15 \%$ 
International Journal of Next-Generation Networks (IJNGN) Vol.6, No.2, June 2014

In all the cases probability of false alarm will also be taken into account. These results will be formulated and communicated later on.

\section{CONCLUSION:}

The approach was to take the decisions in this paper on the basis of power spectral density of the channel which can be used cognitively to search the available spectral gaps those can be used to new incoming users (SU) thus improving the overall channel's throughput. In this work the energy detection spectrum sensing using FFT within the specified frequency band is performed. It has been shown that how the cognitive radio works dynamically with changing the frequency band from one to another and successfully demonstrated in simulation result. The Additive White Gaussian Noise (AWGN) with the Signal to Noise ratio (SNR) values are taken as $5 \mathrm{~dB}, 15 \mathrm{~dB}$ and Attenuation percentages are 10 and 15 has been used. That is the Spectrum Access in Cognitive Radio demonstrated successfully without interfering with the other frequency bands used by the primary user (PU).

\section{REFERENCES}

[1] Samuel Cheng, 2012, Foundation of Cognitive Radio Systems,

[2] Qing Zhao and Brian M. Sadler "A Survey of Dynamic Spectrum Access", 10535888/07/\$25.00@2007IEEE, MAY 2007

[3] Željko Tabaković “A Survey of Cognitive Radio Systems”, Post and Electronic Communications Agency, Jurišićeva 13, Zagreb, Croatia

[4] S. Haykin, "Cognitive radio: Brain-empowered wireless communications," IEEE J. Select. Areas Commun. vol. 23, no. 2, 2005, pp. 201-220

[5] Ian F. Akyildiz, Won-Yeol Lee, Mehmet C. Vuran, Shantidev Mohanty, "Next generation/dynamic spectrum access/cognitive radio wireless networks: A survey" Broadband and Wireless Networking Laboratory, School of Electrical and Computer Engineering, Georgia Institute of Technology, Atlanta, GA 30332, United States, Received 2 January 2006; accepted 2 May 2006

[6] Alice Crohas, July 2008, "Practical Implementation of a Cognitive Radio System for Dynamic Spectrum Access",

[7] Mohamed Hamid, December 2008 "Dynamic Spectrum Access In Cognitive Radio Networks:Aspects of Mac Layer Sensing”, Blekinge Institute of Technology,

[8] A. Bansal, Ms. R. Mahajan "Building Cognitive Radio System Using Matlab", IJECSE, Vol.1, Number 3, 2012

[9] Linda E. Doyle "Essentials of Cognitive Radio", Trinity College, Dublin

[10] Mansi Subhedar and Gajanan Birajdar (2011), "Spectrum Sensing Techniques in Cognitive Radio Networks: A Survey”, International Journal of Next -Generation Networks, Vol.3, No.2.

[11] Proakis, John G. Digital Communications. McGraw-Hill College, 2000.

[12] Anita Garhwal and Partha Pratim Bhattacharya, December 2011, "A Survey on Dynamic Spectrum Access Techniques For Cognitive Radio", International Journal of Next-Generation Networks (IJNGN) Vol.3, No.4,

[13] Tulika Mehta, Naresh Kumar, Surender S Saini , AprIl- June 2013, "Comparison of Spectrum Sensing Techniques in Cognitive Radio Networks", IJECT Vol. 4, Issue spl- 3,

[14] Thesis report on, "Cognitive Radios-Spectrum Sensing Issues",by Amit Kataria presented to the Faculty of the Graduate School at the University of Missouri-Columbia

[15] FCC (2003), ET Docket No 03-237, Notice of inquiry and notice of proposed Rulemaking

[16] S.Taruna, Bhumika Pahwa, September - 2013 "Simulation of Cognitive Radio Using Periodogram", International Journal of Engineering Research \& Technology (IJERT), Vol. 2 Issue 9, 
Authors

Goutam Ghosh was born in India. He has done B.Tech in Electronics and Communication Engineering and currently pursuing M.Tech in Electronics and Communication Engineering from West Bengal University of Technology. He has 5 years experience in industries and 11 years in academics.

Prasun Das was born in India. He has more than 6 years experience in teaching. Presently he is working as Assistant Professor, Department of Electronics \& Communication Engineering, Bengal Institute of Technology \& Management, Santineketan, West Bengal, India. He has some publications and his area of interests are Image Cryptosystems, Speech Signal Modeling, Modern Communication System Design. He has some professional memberships.

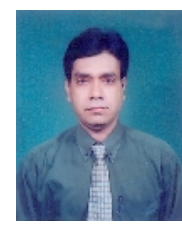

Subhajit Chatterjee was born in India. He has more than 12 years experience in teaching starting and 3 years in industry. He has served some reputed educational institutes in different positions from Lecturer to Teacher In Charge of department. Presently he is working as Assistant Professor, Department of Electronics \& Communication Engineering, Swami Vivekananda Institute of Science and Technology, Kolkata, India. He has number of publications and his area of interests are Analog \& Digital Communications, Solid State Devices,

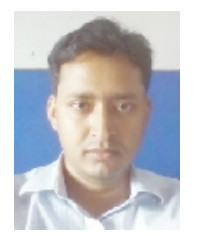

Telecommunication, Microprocessors \& Microcontrollers. He has co authored two books on Microprocessors \& Microcontrollers and Telecommunication \& Switching respectively. He has reviewed chapters of books published by reputed publishers. Presently he is doing his research on Spectrum Access in Cognitive Radio. 